\title{
Vibrational Properties of Disordered Mono- and Bilayers of Physisorbed Sulfur Hexafluoride on $\mathrm{Au}(111)^{\dagger}$
}

\author{
A. W. Rosenbaum, M. A. Freedman, and S. J. Sibener* \\ The James Franck Institute and Department of Chemistry, The University of Chicago, 5640 South Ellis Ave., \\ Chicago, Illinois 60637
}

Received: November 22, 2005; In Final Form: February 6, 2006

\begin{abstract}
We have examined the low-energy single-phonon vibrations of disordered mono- and bilayers of sulfur hexafluoride physisorbed on $\mathrm{Au}(111)$ with inelastic helium atom scattering. At monolayer coverages, $\mathrm{SF}_{6}$ exhibits a dispersionless Einstein mode at $3.6 \pm 0.4 \mathrm{meV}$. We observed two distinct overtones of this vibration as both creation and annihilation events at $7.1 \pm 0.7 \mathrm{meV}$ and $10.9 \pm 1.4 \mathrm{meV}$, respectively. The overtones are harmonic multiples of the fundamental Einstein oscillation. Bilayers of $\mathrm{SF}_{6}$ exhibit a softer fundamental vibration with an excitation energy of $3.3 \pm 0.3 \mathrm{meV}$. This softening, due to the weaker $\mathrm{SF}_{6}$ binding, also results in reduced overtone energies of $6.6 \pm 0.7 \mathrm{meV}$ and $9.8 \pm 0.6 \mathrm{meV}$. The disordered bilayer does not exhibit dispersion, indicating that the molecules are still behaving like Einstein oscillators and not beginning to act as bulk crystalline $\mathrm{SF}_{6}$. The results have improved our understanding of the adsorbate-substrate and interadsorbate interactions which govern the properties of this model molecular physisorption system.
\end{abstract}

\section{Introduction}

Physisorbed noble gases have been studied extensively as model systems in surface science. Rare gases adsorbed on metal surfaces at cryogenic temperatures offer, in particular, key insights into adsorption kinetics, coverage dynamics, adatom diffusion, and crystal growth mechanisms. ${ }^{1-7}$ The octahedral sulfur hexafluoride molecule exhibits many of the inert properties and rotationally averaged symmetry properties of rare gases, and thus represents an experimental adsorption system intermediate between rare gases and complex inorganic or organic thin films. ${ }^{8-13}$

Helium atom scattering (HAS) can probe the adsorbatesubstrate and adsorbate-adsorbate interactions in a nonpertubative manner, giving insight into the state of mono- or multilayers. The deposition of rare gases (Ar, $\mathrm{Xe}$, and $\mathrm{Kr}$ ) on $\mathrm{Ag}(111)$ and $\mathrm{Pt}(111)$ has shown energetic and dispersive trends in phonon modes as a function of film thickness. ${ }^{4,8,14,15}$ In these studies, it was found that a monolayer of a rare gas exhibits a low-energy dispersionless Einstein mode polarized perpendicular to the surface. The energy of this vibration softens, approaching the bulk rare gas frequency, with each additional layer because of the diminishing influence of interactions with the underlying substrate. The bilayer mode not only softens, but a dramatic change in the dispersion plot indicates that the intermolecular forces between the adatoms become increasingly important and alter the character of the vibrational mode observed. As the film thickness increases, the phonon modes continue to systematically approach the band structure of the surface Rayleigh wave of a bulk rare gas crystal. ${ }^{13,16-18}$

In this paper, we examine the vibrational characteristics of mono- and bilayer films of $\mathrm{SF}_{6}$ adsorbed on $\mathrm{Au}(111)$. We do this to better understand the substrate-adsorbate and interadsorbate interactions of sulfur hexafluoride physisorbed on $\mathrm{Au}$ (111) and to examine the scaling properties of the operative

\footnotetext{
† Part of the special issue "John C. Light Festschrift".

* To whom correspondence should be addressed. E-mail: s-sibener@ uchicago.edu.
}

interaction potentials which determine the condensed phase dynamics of this important molecular adsorption system. Numerous studies have examined the adsorption kinetics and structure of $\mathrm{SF}_{6}$, ranging from bulk to physisorbed monolayers. ${ }^{19-24}$ In bulk form, the translational motions of $\mathrm{SF}_{6}$ freeze prior to rotational degrees of freedom, leading to an orientationally disordered bcc crystal from 96 to $223 \mathrm{~K}^{25-27} \mathrm{SF}_{6}$ in thin films or monolayers often does not retain the bulk structure, with structure imposed by the underlying surface. ${ }^{22,23}$ The recent use of $\mathrm{SF}_{6}$ as a dry etching gas has reopened studies into $\mathrm{SF}_{6}$ adsorption. Interest has focused on cryogenic adsorption, negative ion resonance states, ${ }^{28}$ and electron-stimulated desorption. ${ }^{29-31}$ These studies look to elucidate interactions of $\mathrm{SF}_{6}$ with both high-energy electrons and the substrate as critical components to controlling the dry etching processes.

Our studies of the librations of mono- and bilayers of $\mathrm{SF}_{6} /$ $\mathrm{Au}(111)$, in conjunction with the knowledge that comparable rare gas systems show bulk properties at fairly low coverages, could help refine the understanding of heat transfer in bulk molecular crystals. Purskii et al. have studied heat transfer in a variety of simple molecular crystals including bulk $\mathrm{SF}_{6}{ }^{32-34}$ Investigating bulk $\mathrm{SF}_{6}$ from 95 to $220 \mathrm{~K}$, they find that the phonon-phonon coupling term is the dominant component of the thermal resistance of bulk $\mathrm{SF}_{6}$. The phonon-rotation coupling term controls the line shape of the thermal resistance at higher temperatures likely due to an increase in orientational disorder, leading to a decrease in the correlation of neighboring molecular rotations. The thermal conductivity of bulk $\mathrm{SF}_{6}$ approaches the diffusive heat transfer limit, as defined by Cahill et al. on the basis of a simple Einstein oscillator model, indicating that small wave vector vibrations play a key role in heat transfer. ${ }^{35}$ These calculations rely strongly on the determination of the fundamental Einstein oscillations.

\section{Experimental Section}

Experiments were conducted in a high momentum- and energy-resolution helium atom scattering apparatus. Elastic and 
inelastic scattering events are observed through diffraction and time-of-flight (TOF) measurements, respectively. This instrument has been described in detail elsewhere, ${ }^{36,37}$ and its design will only be summarized here. It consists of a cryogenically cooled supersonic helium beam source, an ultrahigh-vacuum (UHV) scattering chamber equipped with appropriate surface characterization tools (such as LEED, AES, etc.), a precollision chopper (chopper-to-ionizer distance of $1.554 \mathrm{~m}$ ), and a rotating, long flight path (sample-to-ionizer distance of $1.005 \mathrm{~m}$ ) quadrupole mass spectrometer detector. The angular collimation yields a resolution of $0.22^{\circ}$, and the $\Delta v / v$ for most beam energies used is less than $1 \%$. The $\mathrm{Au}(111)$ crystal used in these studies was prepared by repeated cycles of sputtering with $0.5 \mathrm{keV} \mathrm{Ne}$ ions followed by annealing above $750 \mathrm{~K}$, until contaminant levels were below our Auger detection limit and helium reflectivity was maximized. Surface crystallinity was confirmed by helium diffraction from the $(23 \times \sqrt{ } 3)$ Au reconstruction ${ }^{38,39}$ with average domain sizes greater than $400 \AA$, with this determination limited by the angular resolution of the apparatus.

Gaseous $\mathrm{SF}_{6}$ (99.8\% purity) was dosed by backfilling the scattering chamber, from a base pressure of $10^{-10}$ Torr to pressures of approximately $10^{-7}$ Torr to achieve varying exposures. Monolayer and submonolayer coverages were created by extended dosing near the overlayer desorption temperature $(\sim 105 \mathrm{~K})$ followed by a quench to the scattering temperature, generally $85 \mathrm{~K}$. $\mathrm{SF}_{6}$ bilayers on $\mathrm{Au}(111)$ were created by dosing at $85 \mathrm{~K}$ for $120 \mathrm{~s}$ at $10^{-7}$ Torr. $\mathrm{SF}_{6}$ coverage was quantified by comparing the Auger peak ratio for sulfur at $152 \mathrm{eV}$ to $\mathrm{Au}$ at $239 \mathrm{eV}$ and through helium atom reflectivity measurements during temperature programmed desorption (TPD). The temperature ramp rate for the TPD studies was $0.1 \mathrm{~K}$ per second.

\section{Results}

Figure 1a shows a typical TPD spectrum for monolayer coverage for a film deposited at $100 \mathrm{~K}$. A similar deposition at $85 \mathrm{~K}$, shown in Figure 1b, followed by a TPD exhibits both the mono- and bilayer desorption features. The bilayer film was prepared using a $120 \mathrm{~s}$ dose at $85 \mathrm{~K}$. Auger electron spectroscopy (AES) was also used to quantify the coverage via the S/Au peak ratio. A prolonged exposure at a surface temperature of $105 \mathrm{~K}$ yields a S/Au Auger peak ratio of 3.75:1. An even longer dose at $85 \mathrm{~K}$ has a S/Au ratio of 7.5:1. The S/Au ratio of the extended low-temperature dose is twice the size of the warmer dose and cannot be increased by successive or longer doses at $85 \mathrm{~K}$ or higher temperatures. The combination of TPD and AES indicate the formation of mono- and bilayers of $\mathrm{SF}_{6}$ and the inability to add a third layer of $\mathrm{SF}_{6}$ because of the high initial sample temperature. After the deposition, time was allowed for the excess $\mathrm{SF}_{6}$ to be evacuated from the chamber. Once the baseline pressure of the scattering chamber was reached, the TPD experiment was started. TPD spectra dosed at $85 \mathrm{~K}$ show two distinct features. The first feature occurs at a surface temperature of $105 \mathrm{~K}$. This increase in signal is the result of bilayer $\mathrm{SF}_{6}$ desorbing from the $\mathrm{SF}_{6} / \mathrm{Au}(111)$ monolayer. The larger change in signal at approximately $110 \mathrm{~K}$ corresponds to the complete desorption of the monolayer. Figure 1a shows a similar experiment with an initial dosing temperature of $100 \mathrm{~K}$ in which only one desorption event is observed during the TPD, indicating monolayer or submonolayer coverage.

Helium atom diffraction along the $\langle 1 \overline{1} 0\rangle$ azimuth was used to characterize the mono- and bilayers of $\mathrm{SF}_{6} / \mathrm{Au}(111)$. Nonspecular features were absent from both systems, suggesting the absence of long-range surface order. Figure 2 shows a

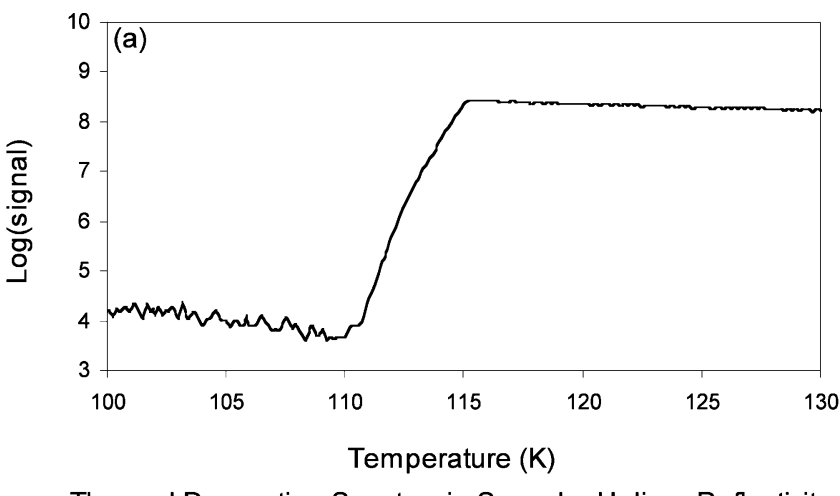

Thermal Desorption Spectra via Specular Helium Reflectivity

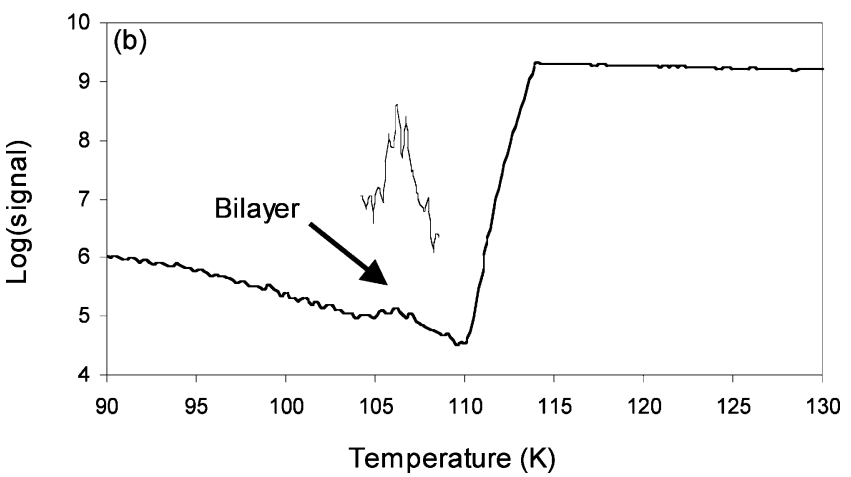

Figure 1. (a) A TPD spectrum taken using specular helium atom reflectivity showing desorption of a monolayer. $\mathrm{SF}_{6}$ was dosed at a background pressure of $10^{-7}$ Torr for $120 \mathrm{~s}$ at a sample temperature of $100 \mathrm{~K}$. Upon completion of the dose, the temperature was ramped through the desorption regime at a rate of $0.1 \mathrm{~K}$ per second. The rapid increase in signal at $110 \mathrm{~K}$ indicates desorption of the monolayer. (b) An example of a TPD spectrum taken using helium atom reflectivity to monitor the surface coverage of $\mathrm{SF}_{6} . \mathrm{SF}_{6}$ was introduced at a sample temperature of $85 \mathrm{~K}$ for $120 \mathrm{~s}$ at a pressure of $10^{-7}$ Torr. After the residual gas was pumped out, the sample temperature was ramped at a rate of $0.1 \mathrm{~K}$ per second through two distinct desorption events. The arrow indicates the bilayer desorption at $105 \mathrm{~K}$. The inset enlarges the bilayer desorption event. Monolayer desorption follows at $110 \mathrm{~K}$ when the signal rapidly recovers to the predose level.

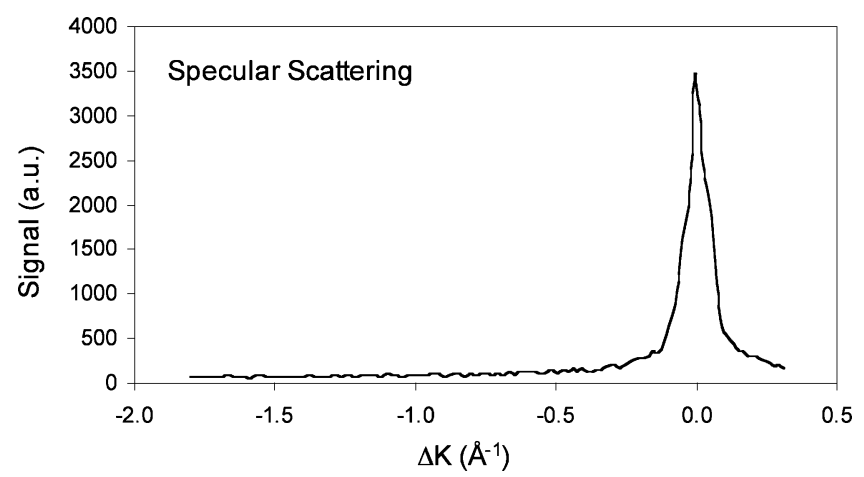

Figure 2. A representative He diffraction scan along the $\langle 1 \overline{1} 0\rangle$ azimuth from a monolayer of $\mathrm{SF}_{6}$ physisorbed on $\mathrm{Au}(111)$. The specular scattering angle was $35.92^{\circ}$ with a beam energy of $22.8 \mathrm{meV}$. In this scan, no subspecular peaks are observed. No superspecular peaks are observed in the complimentary He diffraction scans.

representative diffraction scan taken with a $22.8 \mathrm{meV}$ beam at a specular scattering angle of $35.92^{\circ}$. Low-energy electron diffraction at a range of incident energies, $10-250 \mathrm{eV}$, also showed no indication of long-range adsorbate ordering beyond the underlying $\mathrm{Au}(111)$ spots observed at higher electron beam energies. Additional film preparation, such as annealing near the desorption temperature, did not produce Bragg diffraction rods during $\mathrm{He}$ scattering. We note $\mathrm{X}$-ray and neutron diffraction 

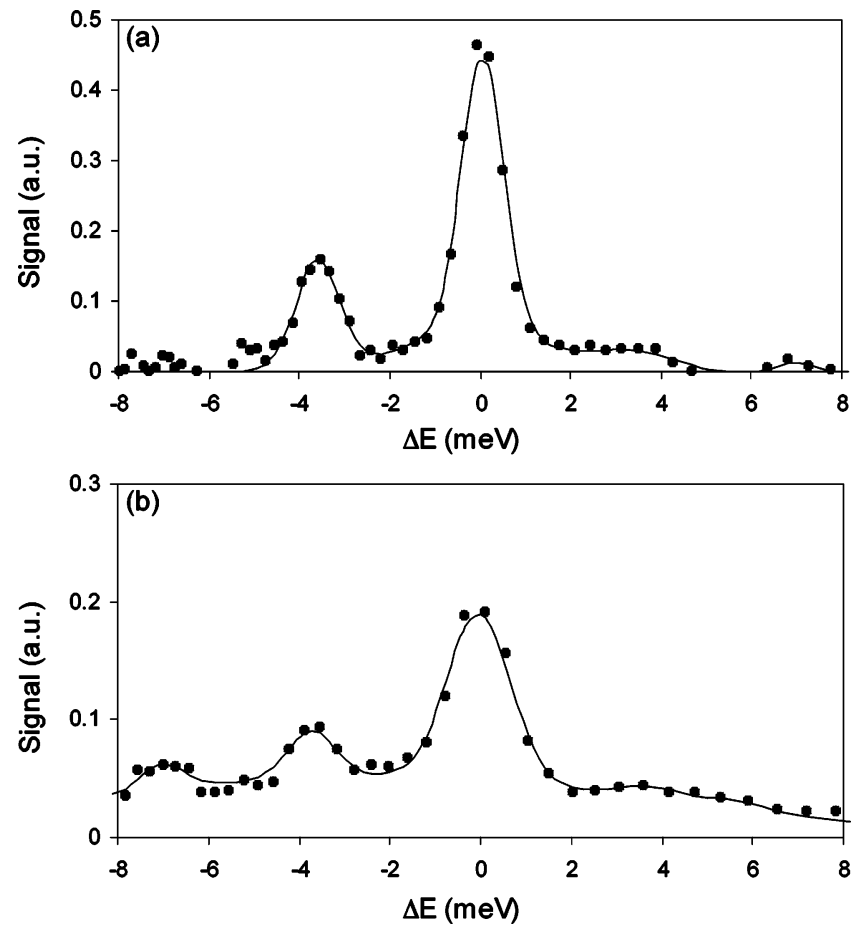

Figure 3. (a) A representative TOF spectrum with a $19.0 \mathrm{meV} \mathrm{He}$ beam shows both creation and annihilation events at $3.6 \mathrm{meV}$ from a monolayer of $\mathrm{SF}_{6}$ at $80 \mathrm{~K}$. The data are shown by the filled circles. The line through the data is a least-squares fit using five Gaussian curves, for the elastic, single, and multiphonon events, and a linear background. The incident and final scattering angles were $35.92^{\circ}$ and $33.92^{\circ}$, respectively. (b) A representative TOF spectrum taken with a $25.5 \mathrm{meV}$ beam showing both the fundamental excitation, $3.6 \mathrm{meV}$, and its harmonic overtone, seen at $7.1 \mathrm{meV}$ from a monolayer of $\mathrm{SF}_{6}$ at $85 \mathrm{~K}$. The data are shown by the filled circles. The line through the data is a least-squares fit using five Gaussian curves, for the elastic, single, and multiphonon events, and a linear background. The incident and final scattering angles are $35.92^{\circ}$ and $37.92^{\circ}$, respectively.

studies found that bulk $\mathrm{SF}_{6}$ forms a bcc crystal above $95 \mathrm{~K}$ and a more complicated monoclinic structure at lower temperatures. ${ }^{25,26} \mathrm{X}$-ray diffraction found monolayer $\mathrm{SF}_{6}$ adsorbed on graphite also undergoes a transformation from a commensurate $(2 \times 2)$ structure below $95 \mathrm{~K}$ to an incommensurate hexagonal structure at higher surface temperatures. ${ }^{22}$

Figure 3 a shows a characteristic TOF spectrum taken with a $19.0 \mathrm{meV}$ He beam scattering from a monolayer of $\mathrm{SF}_{6}$ at a sample temperature of $80 \mathrm{~K}$. The TOF shows both creation and annihilation events associated with the $3.3 \mathrm{meV}$ phonon. For a complete sampling of the phonon modes, it is necessary to vary both beam energy and scattering angles. Higher beam energies will increase the propensity to excite overtones but also diminish the signal-to-noise ratio due to a rise in multiphonon scattering. After sampling from a range of beam energies (15.5-33.4 meV), Figure $3 \mathrm{~b}$ exhibits data collected with the beam energy considered optimal and most frequently used, $25.5 \mathrm{meV}$. Each elastic, inelastic, and multiphonon feature in the inelastic spectra was fit with a Gaussian line shape. The width of the fitted peaks was allowed to vary.

The excitation energy of the fundamental vibration of the $\mathrm{SF}_{6} / \mathrm{Au}(111)$ monolayer remains constant regardless of the scattering condition. Figure 4 shows a dispersion plot ( $\Delta K$ vs $\Delta E$ ) using a variety of beam energies while varying the range of incident angles, from $25.92^{\circ}$ to $35.92^{\circ}$, and final angles, from $26.92^{\circ}$ to $38.92^{\circ}$. Both fundamental annihilation and creation events are seen at sub- and superspecular scattering angles. The data obtained for the dispersion plots span $0.6 \AA^{-1}$, which is

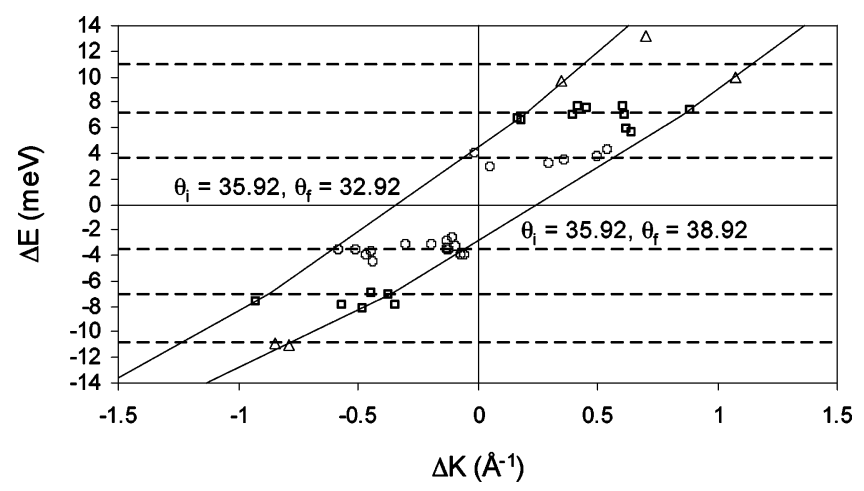

Figure 4. The dispersion plot, $\Delta K$ vs $\Delta E$, for the fundamental phonon and harmonic overtones for monolayer $\mathrm{SF}_{6} / \mathrm{Au}(111)$. Dashed horizontal lines indicate the average creation and annihilation energies. The solid lines running diagonally are two representative scan curves. The data come from a variety of beam energies ranging from 19.0 to $33.5 \mathrm{meV}$ and incident angles from $35.92^{\circ}$ to $25.92^{\circ}$ with exit angles from $38.92^{\circ}$ to $24.92^{\circ}$. Circles, squares, and triangles represent the fundamental, first overtone, and second overtone events, respectively.

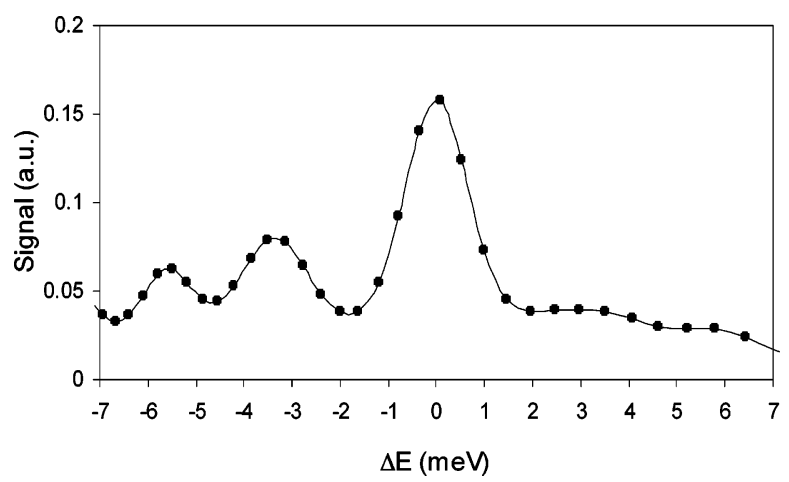

Figure 5. A representative TOF spectrum taken from bilayer $\mathrm{SF}_{6}$ at a surface temperature of $85 \mathrm{~K}$ with a $25.5 \mathrm{meV}$ beam. The fundamental and harmonic excitations of the bilayer occur at $3.3 \pm 0.3 \mathrm{meV}$ and $6.6 \pm 0.7 \mathrm{meV}$, respectively. The data are shown by the filled circles. The line through the data is a least-squares fit using six Gaussian curves, for the elastic, single, and multiphonon events, and a linear background. The incident and final scattering angles are $35.92^{\circ}$ and $37.92^{\circ}$, respectively.

approximately half the distance to the zone edges of $\mathrm{Au}(111)$ of 1.452 and $1.257 \AA^{-1}$ and greater than half the distance for bulk crystalline $\mathrm{SF}_{6}$ below $94 \mathrm{~K}^{25}$

At higher beam energies, we excite more phonons, as shown in Figure 3b. The higher-energy phonon, seen at $7.1 \mathrm{meV}$ in both creation and annihilation events, is the first harmonic overtone. This overtone is found in a large number of spectra, while the second overtone, seen at $10.9 \mathrm{meV}$, is often masked by multiphonon scattering. The dispersion plot for the overtones is shown in Figure 4.

Inelastic scattering from a bilayer of $\mathrm{SF}_{6} / \mathrm{Au}(111)$ taken with a $25.5 \mathrm{meV}$ beam is shown in Figure 5. The fundamental annihilation and creation events are shifted to lower energies, which in turn reduce the excitation energy of the overtones. The phonon remains dispersionless with harmonic overtones seen at 6.6 and $9.8 \mathrm{meV}$, as shown in Figure 6. The data obtained for this dispersion plot again span approximately 0.6 $\AA^{-1}$. Because of the low signal and the multiphonon background, the uncertainties in the peak positions of the overtone modes are greater than for the fundamental modes for both the monoand bilayer systems. These uncertainties fluctuate randomly as seen in the dispersion plots, which indicates that no dispersion is occurring. 


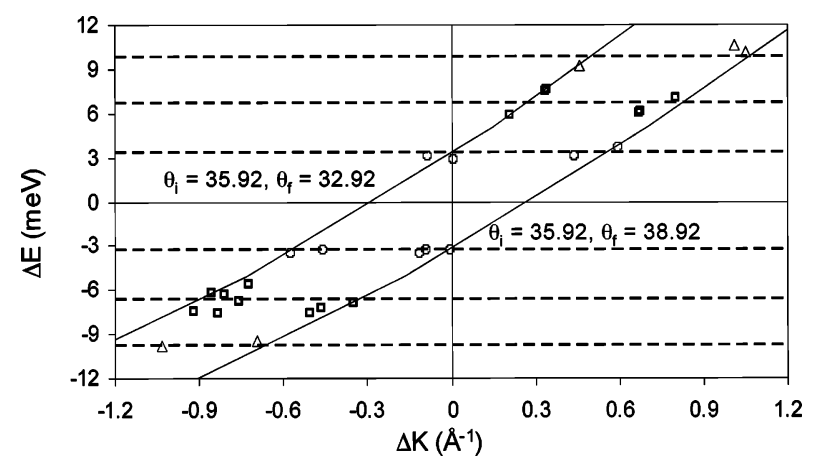

Figure 6. The dispersion plot, $\Delta K$ vs $\Delta E$, for the fundamental and harmonic overtones for bilayer $\mathrm{SF}_{6} / \mathrm{Au}(111)$. Dashed horizontal lines indicate the average creation and annihilation energies. The solid lines running diagonally are two representative scan curves. The data shown come from a variety of beam energies ranging from 19.0 to $25.5 \mathrm{meV}$, an incident angle of $35.92^{\circ}$, and final angles from $32.92^{\circ}$ to $38.92^{\circ}$. Circles, squares, and triangles represent the fundamental, first overtone, and second overtone events, respectively.

\section{Discussion}

From the inelastic scattering, we extracted the Debye-Waller factor, $2 W$, over the temperature range used in this study of $\mathrm{SF}_{6} / \mathrm{Au}(111)$. Since both the mono- and bilayers of $\mathrm{SF}_{6} / \mathrm{Au}-$ (111) exhibit Einstein phonons, we can use the following formulation to get the mean square displacements ${ }^{40}$

$$
\left\langle u_{z}{ }^{2}\right\rangle=\left(\frac{\hbar}{2 M \omega}\right) \operatorname{coth}\left(\frac{\hbar \omega}{2 k_{\mathrm{b}} T}\right)
$$

where $M$ is the mass of the vibrating surface, $T$ is the surface temperature, $\hbar$ is Planck's constant, $k_{\mathrm{b}}$ is Boltzmann's constant, $\omega$ is the fundamental frequency of the Einstein vibration, and $\left\langle u_{z}{ }^{2}\right\rangle$ is the surface normal mean square displacement.

Using the fundamental vibrational frequencies for the monoand bilayers, we get mean square displacements at $85 \mathrm{~K}$ of 1.7 $\times 10^{-2}$ and $2.0 \times 10^{-2} \AA^{2}$, respectively. As expected, the bilayer has a wider range of motion, $18 \%$ greater than the monolayer, due to the diminished interaction with the substrate. These displacements can be converted into Debye-Waller factors for a particular surface temperature by accounting for the perpendicular momentum transfer while scattering at specular to eliminate the component from the parallel momentum transfer

$$
2 W=\Delta k_{z}^{2}\left\langle u_{z}^{2}\right\rangle
$$

where $W$ is the Debye-Waller factor, $\Delta k_{z}$ is the perpendicular momentum transfer, and $\left\langle u_{z}{ }^{2}\right\rangle$ is the perpendicular mean square displacement. The slope of the lines prior to desorption in Figure 1a,b can also be used to determine the Debye-Waller factor and thus the surface motion of the mono- and bilayers.

$$
I=I_{0} \mathrm{e}^{-2 W}
$$

Using these slopes and accounting for the scattering conditions, the bilayer has a $16 \%$ greater range of motion than the monolayer. This result agrees with the calculation based on eq 1.

The mono- and bilayer $\mathrm{SF}_{6}$ mean square displacements can be compared with displacements calculated from fundamental vibrational energies at $85 \mathrm{~K}$ for $\mathrm{Ar}, \mathrm{Kr}$, and $\mathrm{Xe}$ monolayers on $\operatorname{Ag}(111)$ of $5.8 \times 10^{-2}, 4.4 \times 10^{-2}$, and $3.0 \times 10^{-2} \AA^{2}$ respectively. $^{2}$ While the comparison involves two different substrates, the interaction terms of rare gases with $\mathrm{Au}$ and $\mathrm{Ag}$ have been well-documented and are within $10 \% .{ }^{41,42} \mathrm{SF}_{6}$ continues the trend of decreasing mean square displacement with increased mass and polarizability. Prior studies indicate that physical adsorption potentials of rare gases adsorbed on metal surfaces have the same shape and are scalable. ${ }^{43}$ A theoretical study using the fundamental vibrational modes, potential well depths, and van der Waals $C_{3}$ coefficients could be used to determine if the adsorption potential for $\mathrm{SF}_{6} / \mathrm{Au}(111)$ has the same shape as physisorbed rare gases on metals. ${ }^{43}$

While the rare gas and $\mathrm{SF}_{6}$ monolayers behave in a similar fashion, the bilayers show significantly different behavior. The rare gas bilayers show significant increases, 30-50\%, in mean square displacement in comparison to their respective monolayers, while the motion of the $\mathrm{SF}_{6}$ bilayer increases only by $16-19 \%$. This difference in displacement can be explained by considering the significantly larger van der Waals $C_{6}$ coefficient and polarizability of $\mathrm{SF}_{6}$ when compared to rare gases, as discussed below. ${ }^{5,4-46}$ Luo et al. found that interlayer force constants taken with respect to the substrate-adsorbate interaction define the trend in phonon frequency as a function of film thickness. ${ }^{15}$ The smaller change in $\mathrm{SF}_{6} / \mathrm{Au}(111)$ vibrational energy, and thus displacement, from mono- to bilayer is simply a function of the increased interaction term between layers as compared to that of rare gases.

To understand the reduced mobility of the bilayer of $\mathrm{SF}_{6}$ compared to noble gases, the reduced potential curves can be compared. The reduced Morse potential, which can be used around the equilibrium internuclear distance $r_{\mathrm{m}}$, is given by

$$
V(x)=\exp [-2 \beta(x-1)]-2 \exp [-\beta(x-1)]
$$

where $\beta$ is the ratio of the Morse potential parameter and $r_{\mathrm{m}}$, and $x$ is the ratio of the internuclear separation and $r_{\mathrm{m}}$. Barker et al. showed that the rare gas dimer potentials have similar reduced parameters, indicating that the reduced potentials fall on a universal curve. ${ }^{47}$ While $\mathrm{SF}_{6}$ dimers have a smaller well depth $\epsilon$ and larger internuclear separation $r_{\mathrm{m}}$ than $\mathrm{Xe}_{2}$, the curvature of the potential well is such that the reduced potential of an $\mathrm{SF}_{6}$ dimer coincides with the rare gases $\left(\mathrm{He}_{2}, \mathrm{Ne}_{2}, \mathrm{Ar}_{2}\right.$, $\left.\mathrm{Kr}_{2}, \mathrm{Xe}_{2}\right){ }^{48,49}$ At larger internuclear separations where the potential is dominated by the van der Waals constant $C_{6}$, a deviation from the universal curve appropriate for the rare gas dimers arises. While the van der Waals constant is significantly greater than for the rare gases, ${ }^{46}$ the reduced constant $C_{6}^{*}=C_{6} /$ $\epsilon r_{\mathrm{m}}{ }^{6}$ is much smaller because of the larger internuclear separation. It would therefore not be expected that $\mathrm{SF}_{6}$ should follow the trends observed for rare gases. The increased $C_{6}$ of $\mathrm{SF}_{6}$ should lead to larger long-range interactions, suppressing motion on the surface.

The band structure of the $\mathrm{SF}_{6}$ bilayer also departs from ordered rare gas overlayers. The phonon mode of the bilayer does soften energetically, but there is no indication of the dispersion seen in similar rare gas systems as the coverage increases. ${ }^{4}$ The dispersionless mode indicates that the properties of the bilayer are still far from those of bulk crystalline $\mathrm{SF}_{6}$. The lack of collective motions is due to the disordered underlying layer and keeps the bilayer of $\mathrm{SF}_{6} / \mathrm{Au}(111)$ in the independent oscillator regime. In particular, the underlying disorder removes collective interlayer interactions imperative to dispersion. ${ }^{50}$ Future directions might involve increasing the $\mathrm{SF}_{6}$ coverage systematically with a colder substrate and longer exposure times to track the onset of bulk crystalline surface properties. Subsequent studies might also explore the effects of the molecular environment including the underlying phonon density of states of the substrate via examination of wavevectorresolved line shapes and scattering probabilities. 
In summary, we have identified a dispersionless Einstein mode in disordered mono- and bilayers of $\mathrm{SF}_{6}$ physisorbed on $\mathrm{Au}(111)$. The $\mathrm{SF}_{6}$ adsorbs in a disordered state, showing no Bragg diffraction rods indicative of crystalline order. The fundamental phonon mode and the harmonic overtones for the monolayer are observed at $3.6 \pm 0.4 \mathrm{meV}, 7.1 \pm 0.7 \mathrm{meV}$, and $10.9 \pm 1.4 \mathrm{meV}$, respectively. The fundamental mode then softens to $3.3 \pm 0.3 \mathrm{meV}$ for the second layer as the influence of the substrate is diminished. The overtones for the bilayer, seen at $6.6 \pm 0.7 \mathrm{meV}$ and $9.8 \pm 0.6 \mathrm{meV}$, remain harmonic and dispersionless. While the mode softens for bilayer coverage, it remains dispersionless because of its disorder, leaving the system in an independent oscillator regime. These results have improved our understanding of the adsorbate-substrate and interadsorbate interactions which govern the properties of this model molecular physisorption system.

Acknowledgment. We thank Kevin Gibson for sharing his expertise regarding helium scattering from physisorbed species. This work was primarily supported by the Chemical Sciences, Geosciences and Biosciences Division, Office of Basic Energy Sciences, Office of Science, U.S. Department of Energy, grant DE-FG02-00ER15089. We also acknowledge supplemental infrastructure support from the NSF-Materials Research Science and Engineering Center at the University of Chicago, NSFDMR-0213745. M.A.F. gratefully acknowledges support through a National Science Foundation Graduate Research Fellowship.

\section{References and Notes}

(1) Cohen, P. I.; Unguris, J.; Webb, M. B. Surf. Sci. 1976, 58, 429.

(2) Gibson, K. D.; Sibener, S. J. J. Chem. Phys. 1988, 88, 7862.

(3) Gibson, K. D.; Cerjan, C.; Light, J. C.; Sibener, S. J. J. Chem. Phys. 1988, 88, 7911 .

(4) Gibson, K. D.; Sibener, S. J. J. Chem. Phys. 1988, 88, 7893.

(5) Bruch, L. W.; Cole, M. W.; Zaremba, E. Physical Adsorption: Forces and Phenomena; Oxford University Press, Inc.: New York, 1997 Vol. 33.

(6) Kern, K.; Zeppenfeld, P.; David, R.; Comsa, G. Phys. Rev. B 1987, 35,886 .

(7) Siber, A.; Gumhalter, B.; Braun, J.; Graham, A. P.; Bertino, M F.; Toennies, J. P.; Fuhrmann, D.; Woll, C. Phys. Rev. B 1999, 59, 5898 (8) Kern, K.; David, R.; Palmer, R. E.; Comsa, G. Phys. Rev. Lett. 1986, 56,2823

(9) Rosenbaum, A. W.; Freedman, M. A.; Darling, S. B.; Popova, I.; Sibener, S. J. J. Chem. Phys. 2004, 120, 3880.

(10) Darling, S. B.; Rosenbaum, A. W.; Sibener, S. J. Surf. Sci. 2001, 478, L313.

(11) Darling, S. B.; Rosenbaum, A. W.; Wang, Y.; Sibener, S. J. Langmuir 2002, 18, 7462 .

(12) Self-Assembled Monolayers of Thiols; Ulman, A., Ed.; Academic Press: San Diego, CA, 1998; Vol. 24.
(13) Gibson, K. D.; Sibener, S. J. Phys. Rev. Lett. 1985, 55, 1514.

(14) Cardini, G. G.; O'Shea, S. F.; Marchese, M.; Klein, M. L. Phys. Rev. B 1985, 32, 4261 . 5051.

(16) Siber, A.; Gumhalter, B.; Graham, A. P.; Toennies, J. P. Phys. Rev. B 2001, 63, 115411 .

(17) Gibson, K. D.; Sibener, S. J.; Hall, B. M.; Mills, D. L.; Black, J. E. J. Chem. Phys. 1985, 83, 4256 .

(18) Gibson, K. D.; Sibener, S. J. Faraday Discuss. Chem. Soc. 1985, $80,203$.

(19) Klekamp, A.; Umbach, E. Chem. Phys. Lett. 1990, 171, 233.

(20) Taylor, J. C.; Waugh, A. B. J. Solid State Chem. 1976, 18, 241

(21) Klekamp, A.; Umbach, E. Surf. Sci. 1991, 249, 75.

(22) Marti, C.; Ceva, T.; Croset, B.; Beauvais, C. D.; Thomy, A. J. Phys. (Paris) 1986, 47, 1517

(23) Bouchdoug, M.; Ceva, T.; Marti, C.; Menaucourt, J.; Thomy, A. Surf. Sci. 1985, 162, 426 .

(24) Fisher, G. B.; Erikson, N. E.; Madey, T. E.; Yates, J. T. Surf. Sci. 1977, 65, 210.

(25) Isakina, A. P.; Prokhvatilov, A. I. Low Temp. Phys. 1993, 19, 142.

(26) Dolling, G.; Powell, B. M.; Sears, V. F. Mol. Phys. 1979, 37, 1859.

(27) Michel, J.; Drifford, M.; Rigny, P. J. Chim. Phys. 1970, 67, 31

(28) Siller, L.; Vanter, N.; Palmer, R. E. Surf. Sci. 2000, 465, 76.

(29) Faradzhev, N. S.; Kusmierek, D. O.; Yakshinskiy, B. V.; Solovev,

S. M.; Madey, T. E. Surf. Sci. 2003, 528, 20.

(30) Faradzhev, N. S.; Kusmierek, D. O.; Yakshinskiy, B. V.; Madey,

T. E. Low Temp. Phys. 2003, 29, 215.

(31) Souda, R.; Gunster, J. Phys. Rev. A 2003, 67, 1.

(32) Purskii, O. I.; Zholonko, N. N.; Konstantinov, V. A. Low Temp Phys. 2000, 26, 278.

(33) Purskii, O. I.; Zholonko, N. N.; Konstantinov, V. A. Low Temp. Phys. 2003, 29, 771.

(34) Purskii, O. I.; Zholonko, N. N. Phys. Solid State 2004, 46, 2015.

(35) Cahill, D. G.; Watson, S. K.; Pohl, R. O. Phys. Rev. B 1992, 46, 6131.

(36) Niu, L.; Koleske, D. D.; Gaspar, D. J.; Sibener, S. J. J. Chem. Phys. 1995, $102,9077$.

(37) Gans, B.; King, S. F.; Knipp, P. A.; Koleske, D. D.; Sibener, S. J. Surf. Sci. 1992, 264, 81.

(38) Harten, U.; Lahee, A. M.; Toennies, J. P.; Wöll, C. Phys. Rev. Lett. 1985, 54, 2619 .

(39) Barth, J. V.; Brune, H.; Ertl, G.; Behm, R. J. Phys. Rev. B 1990, 42, 9307.

(40) Ellis, T. H.; Scoles, G.; Valbusa, U. Chem. Phys. Lett. 1983, 94 247.

(41) Zaremba, E. Surf. Sci. 1985, 151, 91.

(42) Vidali, G.; Ihm, G.; Kim, H.-Y.; Cole, M. W. Surf. Sci. Rep. 1991, $12,133$.

(43) Vidali, G.; Cole, M. W.; Klein, J. R. Phys. Rev. B 1983, 28, 3064

(44) Standard, J. M.; Certain, P. R. J. Chem. Phys. 1985, 83, 3002.

(45) Pack, R. T. J. Phys. Chem. 1982, 86, 2794.

(46) Kumar, A.; Fairley, G. R. G.; Meath, W. J. J. Chem. Phys. 1985, 83,70 .

(47) Barker, J. A.; W., R. O.; Lee, J. K.; Schafer, T. P.; Lee, Y. T. J. Chem. Phys. 1974, 61, 3081.

(48) MacCormack, K. E.; S., W. G. J. Chem. Phys. 1951, 19, 849.

(49) Farrar, J. M.; S., T. P.; Lee, Y. T. AIP Conf. Proc. 1973, 11, 279.

(50) Woll, C. Appl. Phys. A 1991, 53, 377. 